\title{
Participación laboral en Bogotá: cambios en sus determinantes en la década comprendida entre 2005 y $2015^{\star}$
}

\section{Labor participation in Bogotá: \\ changes in their determinants in the decade between 2005 and 2015}

Nelson Manolo Chávez Muñoz**

Héctor Fabio Ríos Hernández****

Orminso Varón Pulido*****

Recibido: 2 de diciembre de 2016

Revisado: 20 de enero de 2017

Aprobado: 3 de febrero de 2017

* Este documento es producto del proyecto de investigación titulado "Cambios en la estructura de la participación laboral en la ciudad de Bogotá en el período 1990-2014”, realizada en la Universidad La Gran Colombia, Facultad de Ciencias Económicas y Administrativas, Programa de Economía.

** Profesor e investigador de la Universidad La Gran Colombia. Economista, magíster en Economía y doctorando en Estudios Sociales. Correo electrónico: nelson.chavez@ugc.edu.co

*** Profesor e investigador de la Universidad La Gran Colombia. Economista, magíster en Economía y doctorando en Estudios Sociales. Correo electrónico: hector.rios@ugc.edu.co

***** Profesor de la Universidad Autónoma de Colombia. Economista, magíster en Economía y doctorando en Gerencia Pública y Política Social. Correo electrónico: orminsovaronpulido@yahoo.es 


\section{Resumen}

Este documento pretende establecer los posibles cambios en los factores que determinan la participación laboral en Bogotá por medio de un análisis comparativo entre los años 2005 y 2015. Para la investigación se realizó una descripción de los principales indicadores del mercado laboral de Bogotá, tales como población, población en edad de trabajar, población económicamente activa, ocupados, desocupados, subempleo objetivo y población inactiva. De manera seguida, se estimaron econométricamente los determinantes de la participación de la fuerza laboral para los dos años de estudio, utilizando para ello modelos tipo Logit. Los resultados obtenidos evidencian que los determinantes que sufren cambios en la participación laboral de un año a otro son las personas casadas, viudas y solteras; se destaca también la reducción de la probabilidad de los estudiantes y de las mujeres que no participan en la fuerza laboral.

Palabras clave: Economía laboral, mercado de trabajo, participación laboral.

Clasificación JEL: J01, J08, J10.

\section{Abstract}

This document intends to establish the possible changes in the factors that determine labor participation in Bogotá, making a comparative analysis for the years 2005 and 2015. For this, a description was made of the main indicators of the labor market of Bogota, such as population, Population of working age, economically active population, employed, unemployed, objective underemployment and inactive population; The labor force participation determinants for the two years of study were estimated econometrically using Logit models. The results show that the determinants that suffer changes in labor participation from one year to another are married, widowed and single; Also highlighting the reduction of the probability of students and women not to participate in the workforce.

Keywords: Labor economy, labor market, labor participation.

Classification JEL: J01, J08, J10. 


\section{Introduction}

Con el propósito de dar continuidad a los esfuerzos investigativos para profundizar en el conocimiento del mercado laboral colombiano, se propone un estudio cuyo objetivo es identificar los cambios en los determinantes de la participación laboral en la ciudad de Bogotá para los años 2005 y 2015. El documento proporciona información valiosa para entender, desde una perspectiva empírica, los factores que determinan la participación laboral en la ciudad, así como los cambios en las probabilidades que se pudieran haber registrado en tales factores durante los últimos años.

En cuanto a la oferta laboral, se estima un modelo econométrico tipo Logit para establecer el efecto de un grupo de variables en la probabilidad de participación en el mercado de trabajo de Bogotá; se utilizó la Encuesta Continua de Hogares (ECH) para 2005 (DANE, 2005) y la Gran Encuesta Integrada de Hogares (GEIH) para 2015 (DANE, 2015). Aunque este tipo de estimaciones ya se ha realizado para Colombia (véase sección de antecedentes), es pertinente replicar este ejercicio para la ciudad de Bogotá, infiriendo sobre estimadores actualizados de los determinantes de la fuerza laboral en esta ciudad, así como sus posibles cambios en sus probabilidades.

El documento consta, en primer lugar, de una revisión de literatura, la cual comprende el marco teórico que rodea el tema de la oferta de trabajo, seguido de los antecedentes. Posteriormente, se muestran algunos hechos estilizados del mercado laboral bogotano que permiten caracterizarlo en los años 2005 y 2015. Finalmente, se muestran los resultados de los modelos econométricos y las conclusiones a manera de discusión.

\section{Aproximación al marco teórico}

En el tema de participación laboral, se deben tener en cuenta las tendencias demográficas, así como las condiciones económicas y sociales, ya que estas permiten explicar los comportamientos generales del mercado de trabajo. Siguiendo a McConnel y Brue (2003), en el núcleo familiar ha dejado de participar solamente el jefe de hogar en el mercado laboral y se ha observado que ahora son por lo menos dos personas las que trabajan en la familia.

Por su parte, la oferta laboral - o como la llaman los autores en mención, servicios totales de trabajo- está determinada por los siguientes factores: a) la cantidad de nacimientos, muertes e inmigración neta, los cuales componen la dimensión y la estructura demográfica de la población; b) la tasa de participación laboral, que es el número de personas en edad de trabajar que trabaja o está buscando trabajo; c) el número de horas de la jornada de trabajo; y, por último, d) la calidad del trabajo, representada por el nivel de educación y de experiencia de la fuerza laboral (inversión de la población en capital 
humano). De acuerdo con los determinantes de la oferta laboral, este estudio va a enfatizar en el segundo determinante, es decir, en los cambios en la participación laboral que expliquen desde esta perspectiva el comportamiento de la oferta de trabajo tanto de hombres como para mujeres.

Figura 1. Oferta de trabajo del individuo

Panel a

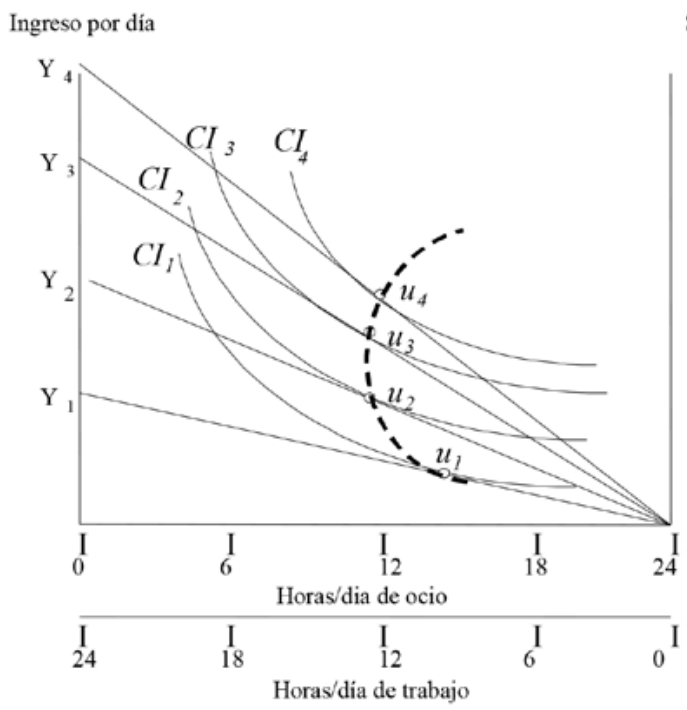

Panel b

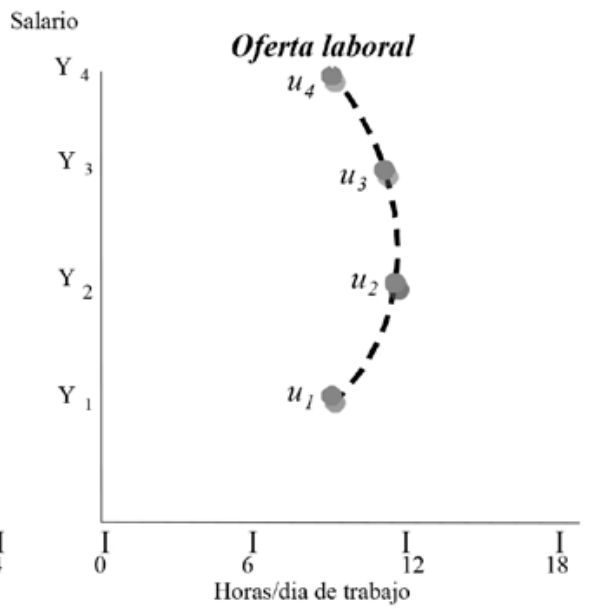

Fuente: elaboración propia con base en McConnel y Brue (2003).

En la figura 2, panel a, se muestran las elecciones óptimas del individuo $\left(\mathrm{u}_{1}, \mathrm{u}_{2}, \mathrm{u}_{3} \mathrm{y} \mathrm{u}_{4}\right)$ de acuerdo con cuatro restricciones presupuestarias $\left(\mathrm{Y}_{1}, \mathrm{Y}_{2}, \mathrm{Y}_{3}\right.$ y $\left.\mathrm{Y} 4\right)$ y cuatro curvas de indiferencia $\left(\mathrm{CI}_{1}, \mathrm{CI}_{2}, \mathrm{CI}_{3}\right.$ y $\left.\mathrm{CI}_{4}\right)$. Si el trabajador se encuentra en una situación inicial en la que la elección óptima es $\mathrm{u}_{1}$ y se registra un incremento de su salario, entonces, la recta presupuestaria se desplaza de $\mathrm{Y}_{1}$ a $\mathrm{Y}_{2}$, la curva de indiferencia se desplaza de $\mathrm{CI}_{1}$ a $\mathrm{CI}_{2}$ y se alcanza un nuevo punto de equilibrio en $\mathrm{u}_{2}$. Este comportamiento es explicado porque el incremento del salario incentiva al trabajador a aumentar las horas destinadas al trabajo por día y, por lo tanto, a reducir las horas diarias destinadas al ocio. Igualmente ocurre si sigue aumentando el ingreso de $\mathrm{Y}_{2}$ a $\mathrm{Y}_{3}$, sin embargo, cuando el ingreso se incrementa de $\mathrm{Y}_{3}$ a $\mathrm{Y}_{4}$, tanto la restricción presupuestaria como la curva de indiferencia se desplazan de $\mathrm{Y}_{3}$ a $\mathrm{Y}_{4}$ y de $\mathrm{CI}_{3}$ a $\mathrm{CI}_{4}$, respectivamente, generándose un nuevo óptimo en $\mathrm{u}_{4}$, y esta elección se traduce en una menor cantidad de horas destinadas al trabajo y, por consiguiente, en una mayor cantidad de horas destinadas al ocio. Con respecto al análisis anterior, se puede afirmar que el tiempo que una persona destina al trabajo aumenta conforme también lo hace el salario, sin embargo, existe un punto en el cual, si el 
salario se sigue incrementando, dicha persona reduce las horas de trabajo y aumenta las de ocio, reduciéndose, por lo tanto, su oferta laboral. Este comportamiento individual es explicado por el efecto renta y el efecto sustitución. El efecto renta determina el número de horas que el individuo desea trabajar una vez varía su renta, manteniéndose el salario constante, lo cual se traduce en que, si la renta aumentara cada vez más, la persona estaría dispuesta a destinar más horas al ocio y menos horas al trabajo. Por su parte, el efecto sustitución se refiere a cómo una variación del salario, manteniéndose constante la renta, genera variaciones en las horas destinadas al trabajo, es decir, si el salario fuera cada vez mayor, la persona estaría dispuesta a destinar más horas al trabajo que al ocio, por lo cual sustituiría horas de ocio por horas de trabajo. En este sentido, el efecto neto depende de las preferencias del individuo entre las horas destinadas al trabajo y las horas destinadas al ocio, por lo tanto, si el efecto renta domina al efecto sustitución, un incremento del salario incentiva al trabajador a laborar menos horas, por lo que la curva de oferta laboral tiene pendiente negativa. Por su parte, si el efecto sustitución es dominante frente al efecto renta, un aumento del salario incentiva al individuo a trabajar más horas y, de esta manera, la pendiente de la curva de oferta laboral es positiva.

\section{Antecedentes}

Se presenta a continuación la revisión de literatura que contempla los trabajos que anteceden a la presente investigación.

Arango y Posada (2001) realizaron un estudio en el que analizaron, en primer lugar, cómo evolucionó el desempleo urbano en Colombia para los años comprendidos entre 1984 y 2000; dicho estudio también analizó si efectivamente la tendencia de la tasa de desempleo creció a un mayor ritmo que la tasa natural de desempleo. Por consiguiente, los autores analizaron las propiedades de las series temporales para variables principales del mercado de trabajo, como tasa de ocupación, tasa de desempleo y tasa de participación, tanto a nivel nacional como por ciudades. Los autores concluyeron que la tasa de desempleo urbana en Colombia no ha sido estacionaria y ha presentado un comportamiento en forma de "u", reduciéndose en los años 1984 y 1994, para luego incrementarse hasta el año 2000.

Arango y Posada (2003) aportan al debate de los determinantes de la tasa de participación laboral para las siete ciudades de Colombia, utilizando para ello la Encuesta Nacional de Hogares para el período 1984 a 2000. Dichos determinantes se estimaron para cuatro categorías: mujeres comprometidas, mujeres no comprometidas, hombres comprometidos y hombres no comprometidos. Se realizaron estimaciones econométricas en las cuales la variable explicada fue de orden binario, que representaba la participación o no en el mercado laboral; por su parte, las variables explicativas fueron educación, niños 
menores de seis años, riqueza, otros desempleados en el hogar, edad, edad al cuadrado y dos variables dummies apropiadas. El documento concluye que existen diferencias entre los diferentes grupos o categorías establecidas. De esta manera, según su estudio, los factores que incrementan la participación laboral son el desempleo de los otros miembros del hogar, el nivel de educación y la edad, mientras que la variable que reduce la participación laboral es la riqueza de los hogares. Finalmente, si la tasa de desempleo disminuye, entonces la tasa de participación también lo hace.

Arango, Posada y Charry (2003) realizaron una extensión al estudio de los determinantes de la participación laboral para Colombia - reseñado anteriormente-, utilizando ahora la Encuesta Continua de Hogares para trece ciudades de Colombia para los años 2000 a 2002. Se compararon los resultados de ambos estudios. Los modelos econométricos estimados fueron Probits, permitiendo calcular de esta manera los efectos marginales de cada una de las variables explicativas. La conclusión principal del estudio es que los determinantes que tienen un efecto positivo y negativo en la participación laboral no cambian con la Encuesta Nacional de Hogares ni con la Encuesta Continua de Hogares.

Romero (2006) estimó un modelo Probit para determinar la probabilidad de que una persona participe en el mercado laboral, cuya variable dependiente es dicotómica, tomando valor de uno si participa en el mercado laboral y valor de cero si no lo hace. Las variables que explican la participación laboral tanto para hombres como para mujeres para la ciudad de Bogotá se determinaron por medio de la Encuesta Continua de Hogares. De esta manera, el documento concluye, para los hombres, que la decisión de participar en el mercado laboral está determinada por la edad, la tasa de desempleo de otros miembros del hogar, el subempleo para los jefes de hogar y el nivel educativo, mientras que la edad al cuadrado reduce la participación laboral, al igual que la asistencia escolar. Por su parte, para las mujeres, la edad, la asistencia escolar para hija soltera, la tasa de desempleo de otros miembros del hogar y el nivel educativo aumentan la probabilidad de participar en el mercado de trabajo, mientras que las hijas solteras y casadas reducen dicha participación de las mujeres.

Por su parte, Chávez, Velásquez y Mateus (2011) realizaron un estudio en el que identificaron los cambios estructurales en los determinantes de la participación en el mercado laboral entre los diferentes grupos poblacionales que explican el comportamiento de la oferta laboral en Colombia para el período comprendido entre 1984 y 2008. La metodología consistió en estimar un modelo econométrico Probit de elección binaria, y se realizaron las pruebas de Cusum y Chow con el fin de establecer si hubo cambios estructurales en la participación laboral en el país. Los autores concluyeron que la tasa de crecimiento económico aumenta la participación laboral, mientras que la educación, la edad, la edad al cuadrado y la asistencia escolar la reducen. Finalmente se argumentó que en 1999 hubo un cambio estructural en los factores que determinan la participación en el mercado laboral, debido, principalmente, a la recesión económica presentada en 
este año, es decir, que la participación laboral se incrementó en este período, independientemente de los factores que inicialmente aumentaron dicha participación.

\section{Hechos estilizados}

En esta parte del estudio se describirá el análisis realizado de la estructura del mercado laboral para la ciudad de Bogotá entre los períodos 2005 y 2015. Cabe anotar que las cifras fueron tomadas del Departamento Administrativo Nacional de Estadística (DANE).

Para poder analizar los cambios en los determinantes del mercado laboral, es necesario tomar un espacio de tiempo suficiente para establecer si las políticas y las condiciones económicas han cambiado o si, por el contrario, esta estructura se mantiene sin cambios a través de los años. Para facilitar el análisis, se evalúan, en primera instancia, los principales cambios demográficos (Alarco, Lora y Orellana, 1990) presentados en la población bogotana para el período de estudio. En segunda instancia, se analizan (Alarco et al., 1990) los cambios de los indicadores del mercado laboral en la última década en la ciudad de Bogotá.

\subsection{Análisis demográfico}

La población de la ciudad de Bogotá en la última década ha sufrido un incremento significativo, pasando de 6824000 habitantes en 2005 a 7862242 habitantes en 2015. Esto representa un incremento de la población del 15,2 \% en la última década.

Figura 2. Evolución de la población bogotana 2005-2015

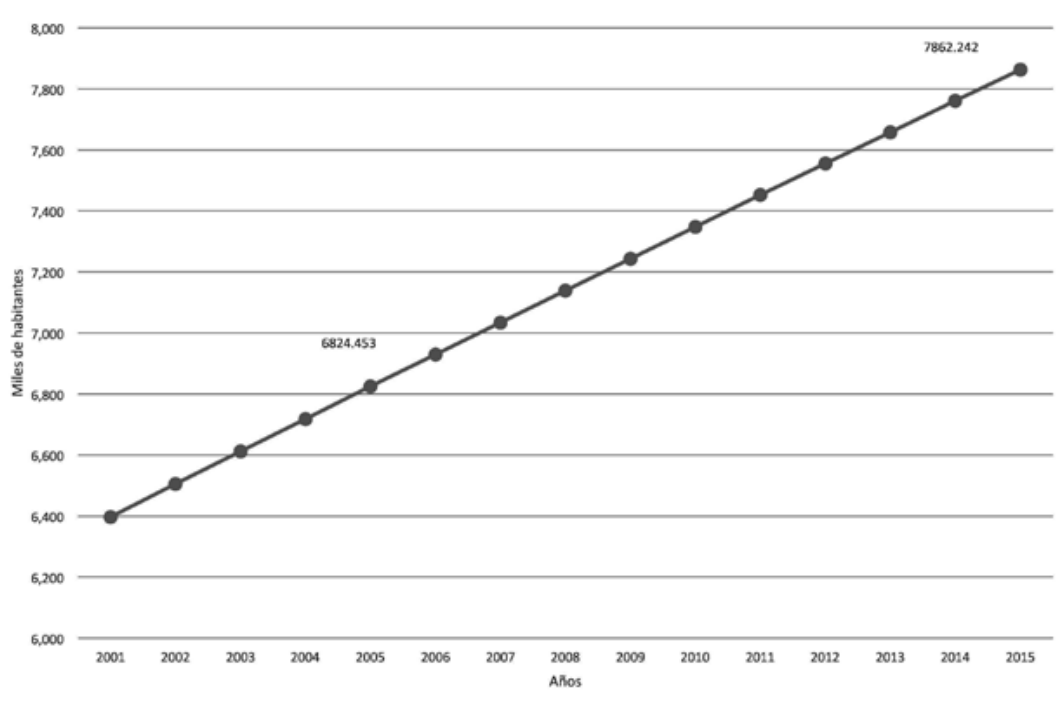

Fuente: elaboración propia con base en datos proporcionados por el DANE. 
Por otra parte, la población en edad de trabajar $\left(\mathrm{PET}^{1}\right)$ en los períodos de análisis pasó de un total de 5354425 personas a 6425103 individuos, de manera que se registró un incremento en términos poblacionales del $20 \%$. La figura 3 muestra la evolución de este segmento de la población desde 2001 hasta 2015.

Figura 3. Evolución de la población en edad de trabajar en Bogotá 2005-2015

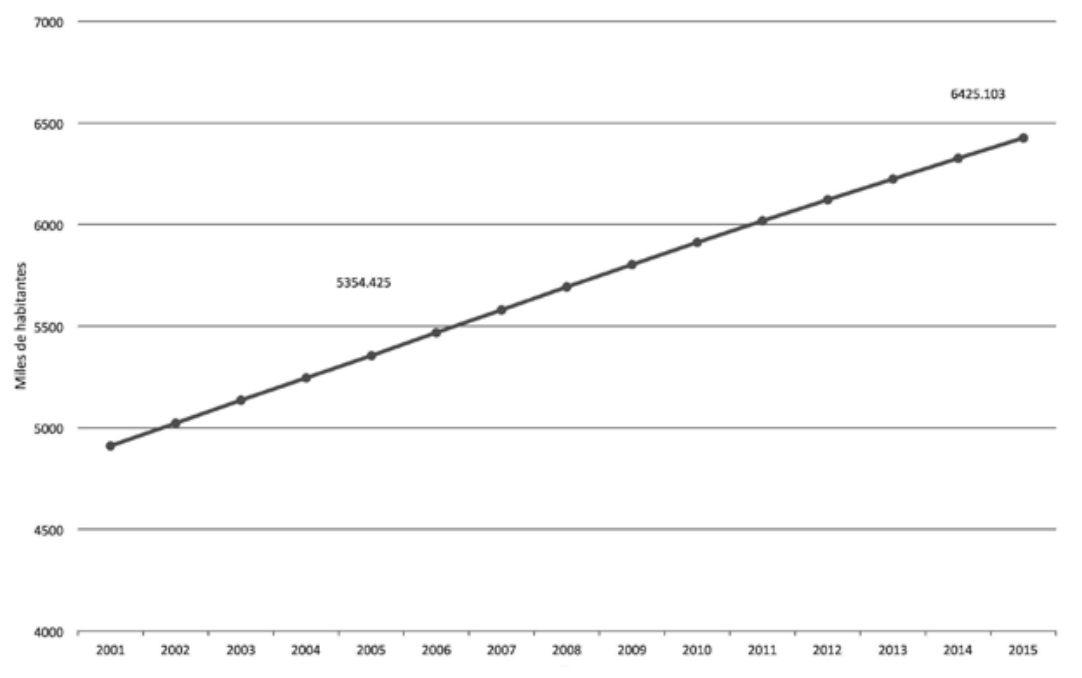

Fuente: elaboración propia con base en datos proporcionados por el DANE.

$\mathrm{Al}$ analizar la fuerza de trabajo o población económicamente activa ${ }^{2}$ (PEA) en el intervalo de tiempo de referencia del estudio, se puede observar que se registró un incremento del 29,4\%, pasando de 3555862 individuos en 2005 a 4601922 personas en 2015. Esto implica un incremento bastante importante en la fuerza de trabajo capitalina, ya que en 10 años la población económicamente activa se incrementó casi un 30 \%.

Por su parte, la PEA está compuesta por las personas ocupadas y las desocupadas. Para poder observar los cambios en la fuerza de trabajo en la capital, es necesario determinar el comportamiento de estos dos componentes. Se presenta a continuación un gráfico que ilustra dicho comportamiento desde 2001.

Como es de esperarse, la tendencia entre ocupados y desocupados debe ser inversa, sin embargo, sorprende el comportamiento registrado en el año 2012, cuando el desempleo y el empleo siguieron una tendencia creciente; esto se pudo deber al crecimiento de la población, que impacta de forma positiva a la PEA y, por ende, a los empleados

1 La PET está compuesta por las personas mayores de 12 años en las zonas urbanas y por las personas mayores a 10 años en las zonas rurales.

2 La PEA para el caso de Bogotá está constituida por las personas mayores de 12 años que se encuentran ocupadas o que están en busca de un empleo. 
y desocupados. Otra explicación factible es el ingreso de los aspirantes, los cuales están iniciando su etapa laboral; generalmente esta población está compuesta por jóvenes recién egresados del colegio o de la universidad.

Figura 4. Evolución de la población económicamente activa en Bogotá 2005-2015

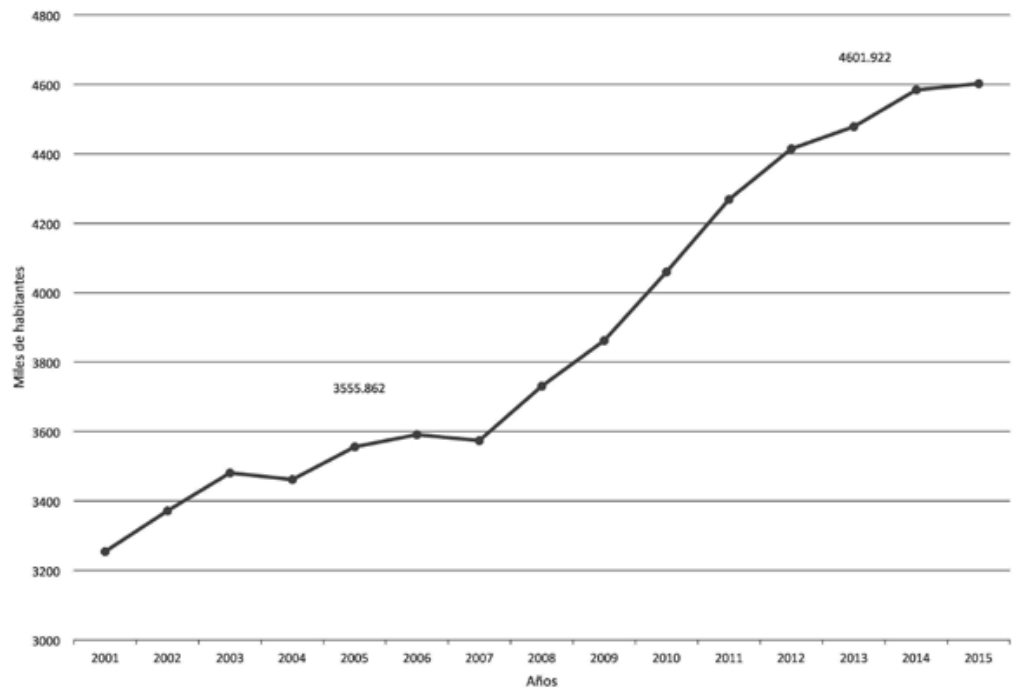

Fuente: elaboración propia con base en datos proporcionados por el DANE.

Figura 5. Evolución de la población ocupada y de la población desocupada en Bogotá 2005-2015

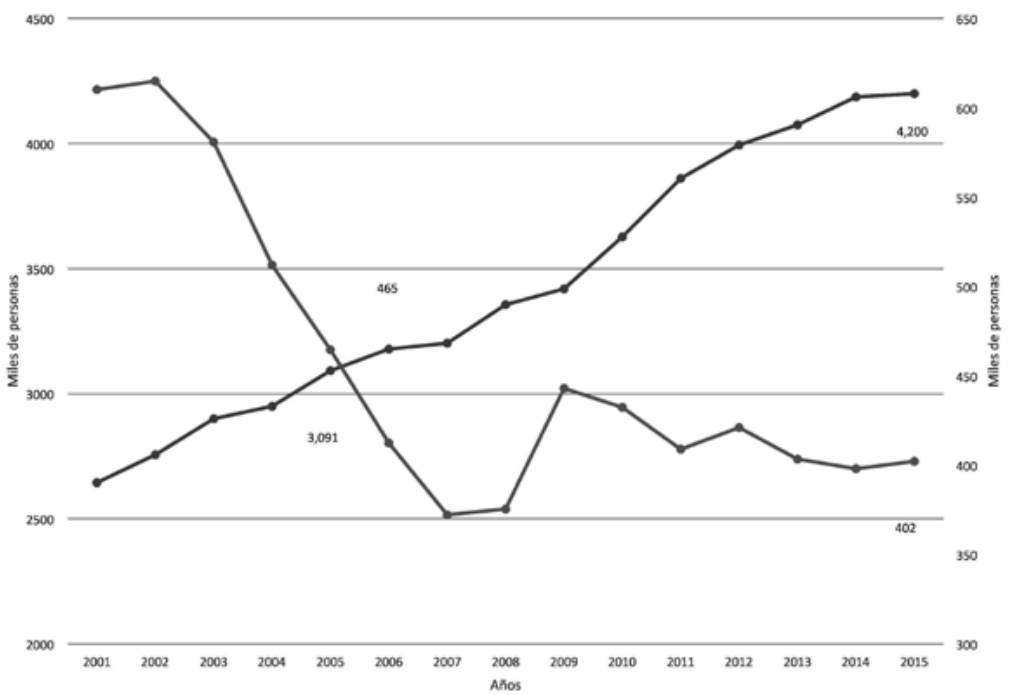

Fuente: elaboración propia con base en datos proporcionados por el DANE. 
$\mathrm{Al}$ analizar con detalle la ocupación en los períodos de referencia, se puede observar que las personas que se encontraban en una situación de subempleo objetivo en 2005 eran 393 804, mientras que en 2015 alcanzaron el número de 514 335. Esto implica un incremento del 30,6\% entre los períodos analizados.

Figura 6. Evolución de la población ocupada en situación de subempleo objetivo en Bogotá 2005-2015

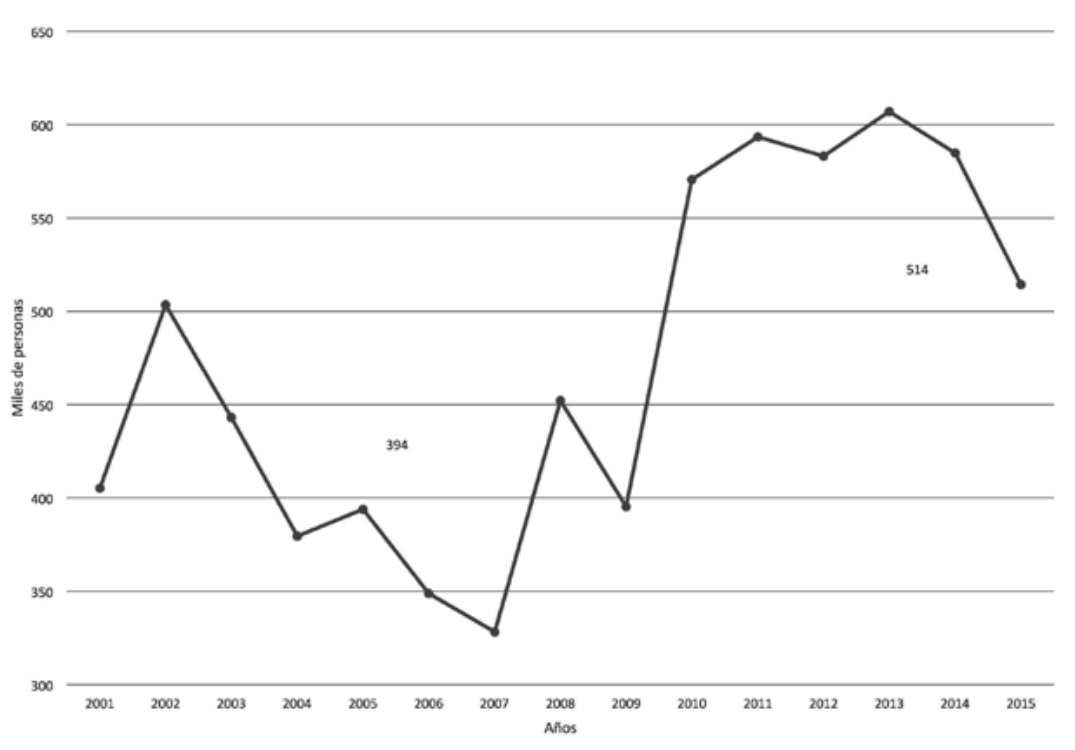

Fuente: elaboración propia con base en datos proporcionados por el DANE.

Sin embargo, al analizar la proporción de subempleados objetivos en relación con el total de ocupados, se puede determinar que el subempleo objetivo disminuyó en cinco puntos básicos, pasando de 12,7 \% en 2005 a 12,2 \% en 2015. Esto pudo ser resultado de las políticas educativas implementadas por el gobierno en la última década, mejorando las condiciones del trabajador.

Tabla 1. Relación de los subempleados objetivos y el total de ocupados en Bogotá 2005-2015

\begin{tabular}{|c|c|}
\hline Año & Proporción \\
\hline 2005 & $12,7 \%$ \\
\hline 2015 & $12,2 \%$ \\
\hline
\end{tabular}

Fuente: elaboración propia con base en datos proporcionados por el DANE. 
Por último, se analizó la población económicamente inactiva $\left(\mathrm{PEI}^{3}\right)$, cuyo comportamiento en el período de referencia presentó un aumento, pasando de 1798563 personas en 2005 a 1823181 en 2015. Esto representa un incremento del 1,4\% entre los períodos analizados.

Figura 7. Evolución de la población inactiva en Bogotá 2005-2015

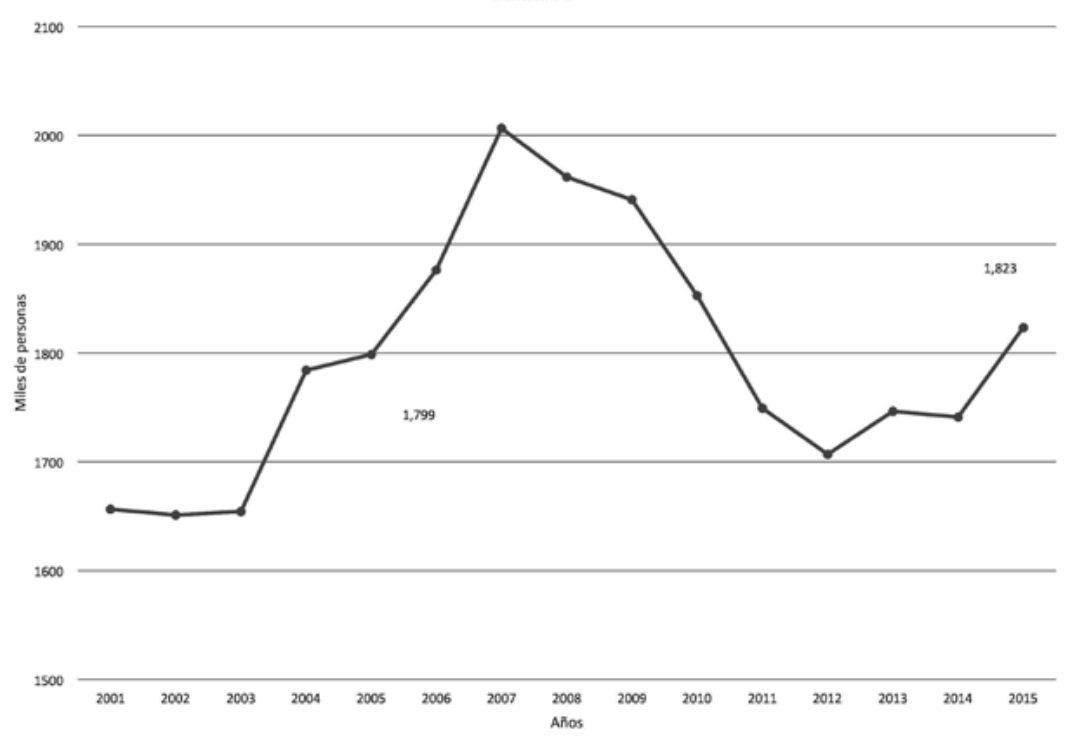

Fuente: elaboración propia con base en datos proporcionados por el DANE.

La proporción de la población inactiva en relación con la población en edad de trabajar, es decir, las personas mayores de 12 años en las zonas urbanas, arrojó los siguientes resultados:

Tabla 2. Proporción de los inactivos en relación con la PET en Bogotá 2005-2015

\begin{tabular}{|c|c|}
\hline Año & Proporción \\
\hline 2005 & $33,6 \%$ \\
\hline 2015 & $28,4 \%$ \\
\hline
\end{tabular}

Fuente: elaboración propia con base en datos proporcionados por el DANE.

$\mathrm{Al}$ analizar los resultados anteriores, se puede determinar que la población inactiva se redujo aproximadamente en 5 puntos porcentuales en la última década, esto se pudo

3 La población económicamente inactiva está compuesta por las personas que no hacen parte de la fuerza laboral (ocupados y desocupados) por distintos motivos, entre los que se encuentran estar dedicados exclusivamente a estudiar, dedicarse únicamente a las labores del hogar, ser solamente pensionados o jubilados, entre otros. 
deber a las mejoras en las condiciones de vida de los habitantes de la ciudad capital y a la inclusión de nuevos trabajadores al mercado.

En la tabla 3 se muestra un resumen de los aspectos demográficos analizados anteriormente.

Tabla 3. Resumen de las principales estadísticas demográficas en Bogotá 2005-2015

\begin{tabular}{|l|c|c|c|}
\hline \multicolumn{1}{|c|}{ Concepto } & \multicolumn{2}{|c|}{ Número de personas } & Variación \% \\
\hline Población total & 6824,453 & 7862,242 & $15,2 \%$ \\
\hline Población en edad de trabajar & 5354,425 & 6425,103 & $20,0 \%$ \\
\hline Población económicamente activa & 3555,862 & 4601,922 & $29,4 \%$ \\
\hline Ocupados & 3091,336 & 4199,726 & $35,9 \%$ \\
\hline Desocupados & 464,526 & 402,196 & $-13,4 \%$ \\
\hline Subempleados objetivos & 393,804 & 514,335 & $30,6 \%$ \\
\hline Inactivos & 1798,563 & 1823,181 & $1,4 \%$ \\
\hline
\end{tabular}

Fuente: elaboración propia con base en datos proporcionados por el DANE.

\subsection{Análisis de los indicadores laborales}

Para realizar un análisis más preciso del mercado laboral en la ciudad de Bogotá, se realizó un comparativo entre los años 2005 y 2015, con el fin de determinar las diferencias en términos relativos de los principales indicadores del mercado laboral en la ciudad. El primer indicador analizado fue el porcentaje de la población en edad de trabajar (\% PET), el cual determina la proporción de la población en edad de trabajar y la población total. Su fórmula de cálculo es la siguiente:

$$
\% P E T=\frac{P E T}{P T}(1)
$$

Los resultados obtenidos se muestran en la figura 8.

$\mathrm{Al}$ analizar los cambios presentes en el porcentaje del PET, se puede determinar que la población en edad de trabajar se incrementó en términos porcentuales 3,3\%, lo cual implica un aumento de la población activa y de la inactiva. Esto se debe principalmente al crecimiento demográfico sufrido por el país en la última década, que es positivo si dicha relación incrementa en mayor proporción la PEA. 
Por su parte, en cuanto al comportamiento de la tasa global de participación ${ }^{4}$ entre los períodos analizados, se observa que entre 2005 y 2015 la participación de la PEA se incrementó $5,2 \%$, lo cual permite deducir que, entre los años analizados, la fuerza laboral ocupó una mayor proporción entre los inactivos, los cuales conforman el otro componente de la PET.

Figura 8. Cambios en el \% del PET en Bogotá 2005-2015

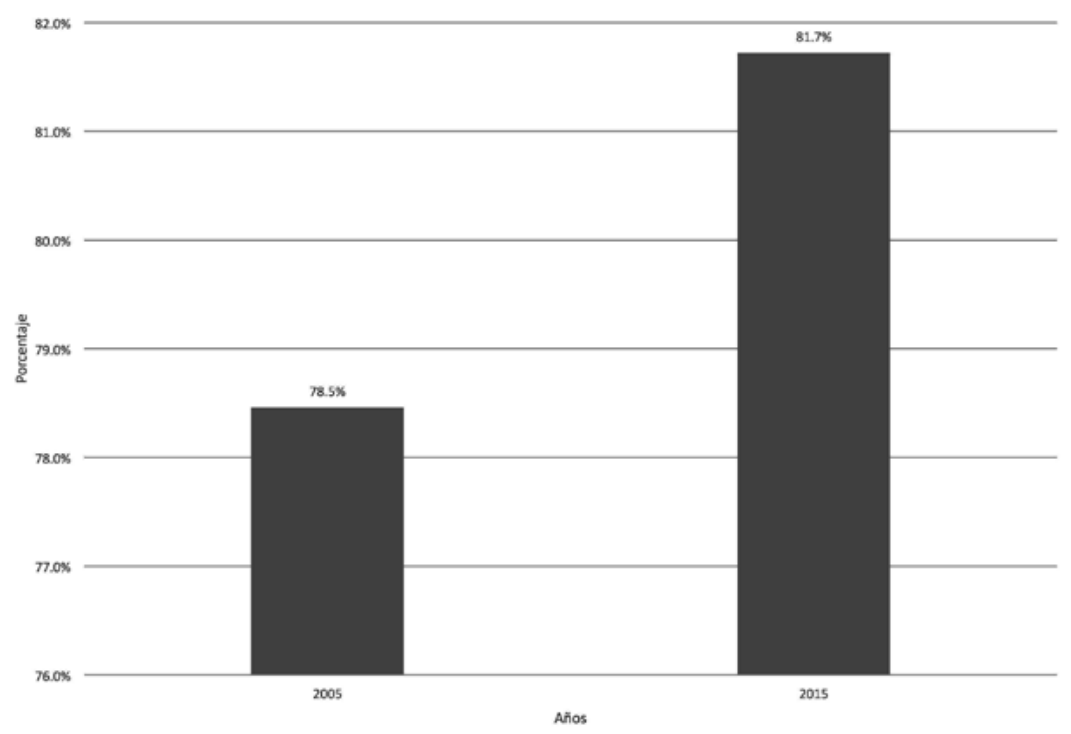

Fuente: elaboración propia con base en datos proporcionados por el DANE.

La tasa de ocupación es al igual una razón, la cual es el cociente entre la población ocupada y la población en edad de trabajar; su fórmula de cálculo es la siguiente:

$$
T O=\frac{O C}{P E T}(2)
$$

En la fórmula, OC corresponde al número de personas ocupadas y PET se refiere a la población en edad de trabajar en el período de referencia.

En 2005, Bogotá presentó una tasa de ocupación del 57,7 \%, proporción menor a la presentada en 2015, cuando la ocupación alcanzó el 65,4 \%, creciendo así un 7,6 \% en esta década. La figura 10 muestra el comparativo de estos dos períodos.

4 Este indicador relaciona la población económicamente activa y la población en edad de trabajar, determinando el porcentaje de la fuerza laboral. Su fórmula de cálculo es la siguiente:

$$
T G P=\frac{P E A}{P E T}
$$


Figura 9. Cambios en la tasa global de participación en Bogotá 2005-2015

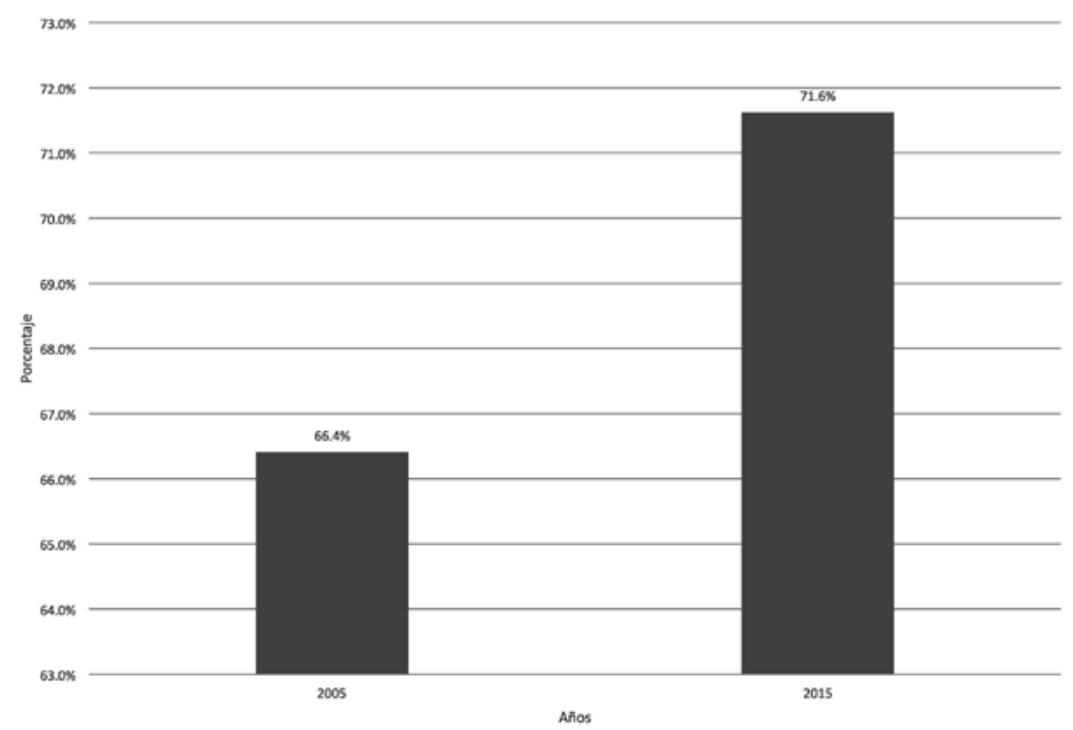

Fuente: elaboración propia con base en datos proporcionados por el DANE.

Figura 10. Cambios en la tasa de ocupación en Bogotá 2005-2015

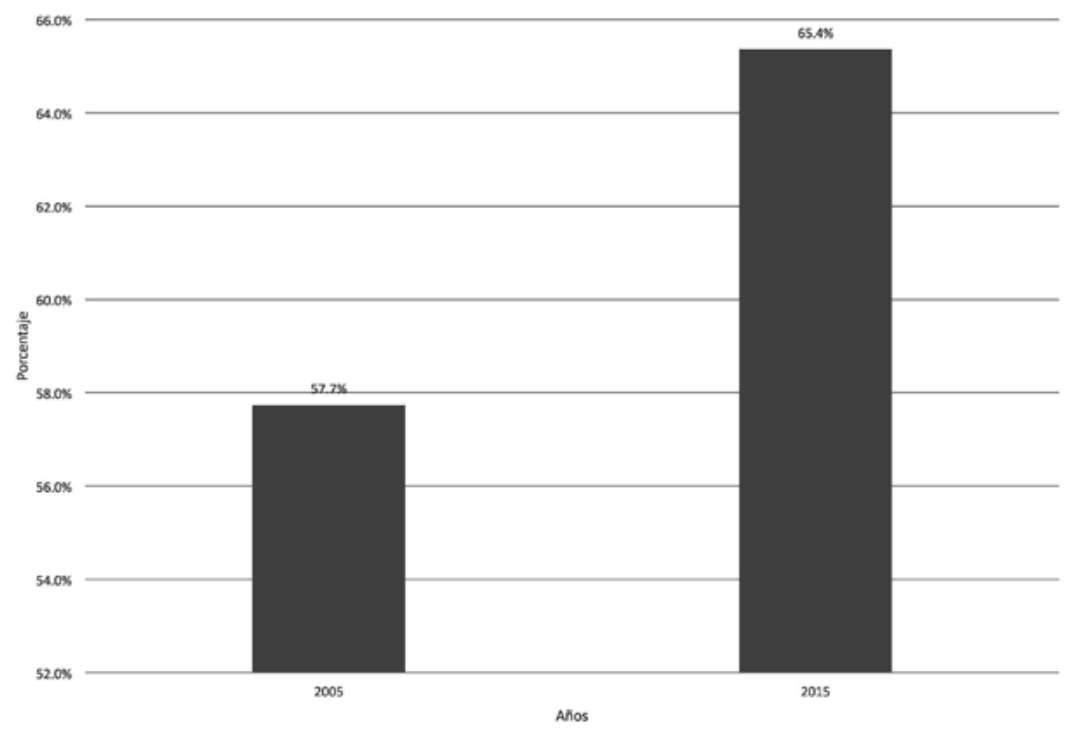

Fuente: elaboración propia con base en datos proporcionados por el DANE.

En Colombia, la tasa de desempleo (TD) es la razón entre el número de desocupados (DS) y la población económicamente activa (PEA); en este sentido, su fórmula general de cálculo es la siguiente: 


$$
T D=\frac{D E S}{P E A}
$$

En el período de análisis, la tasa de desempleo para Bogotá presentó una disminución del 4,4\%, que refleja las mejores condiciones de empleabilidad en la ciudad capital. Este fenómeno se debe principalmente a la estabilidad macroeconómica presentada en el país en la última década, acompañada de una creciente inversión extranjera directa (figura 11).

Figura 11. Cambios en la tasa de desempleo en Bogotá 2005-2015

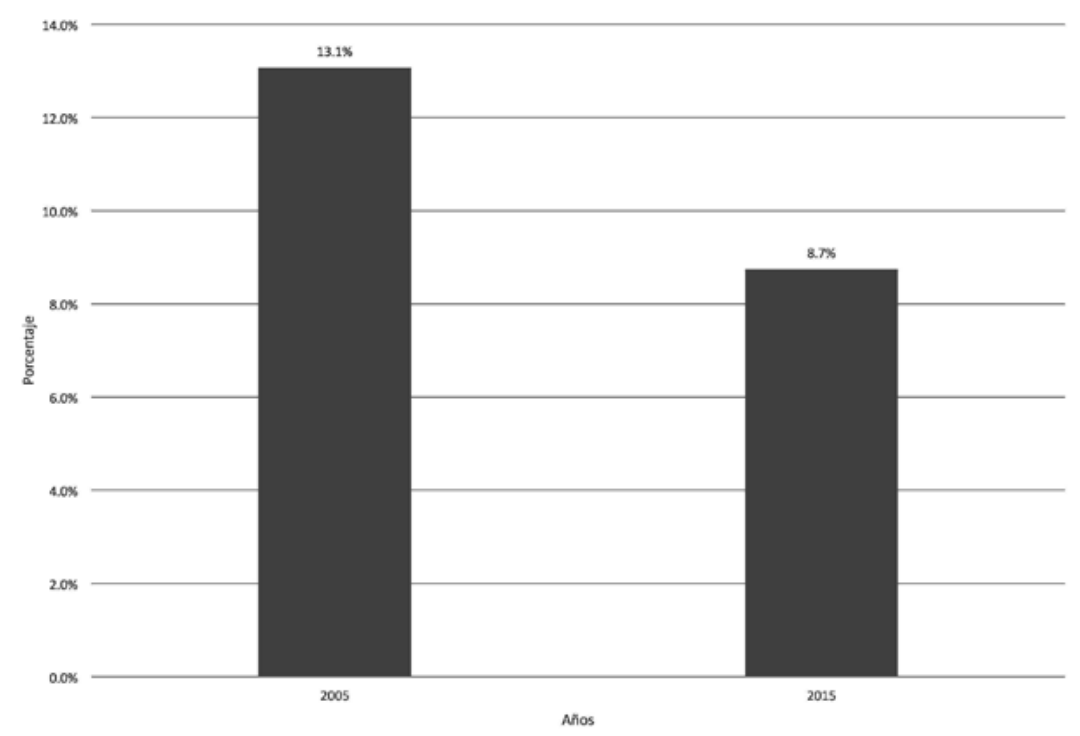

Fuente: elaboración propia con base en datos proporcionados por el DANE.

Por último, se analizó la tasa de subempleo objetivo, la cual es la razón entre la población subempleada (PS) y la población económicamente activa (PEA); su algoritmo de cálculo es el siguiente:

$$
T S=\frac{P S}{P E A}(4)
$$

Para la ciudad de Bogotá, la tasa de subempleo permaneció prácticamente sin cambios en los últimos 10 años, presentándose una diferencia marginal de 0,1\% entre 2005 y 2015. Esto implica que las personas que se encuentran ocupadas no están desempeñando las funciones apropiadas a su competencia, poseen jornadas laborales muy cortas o sus ingresos no son acordes con las funciones realizadas en sus cargos. 
Figura 12. Tasa de subempleo objetivo en Bogotá 2005-2015

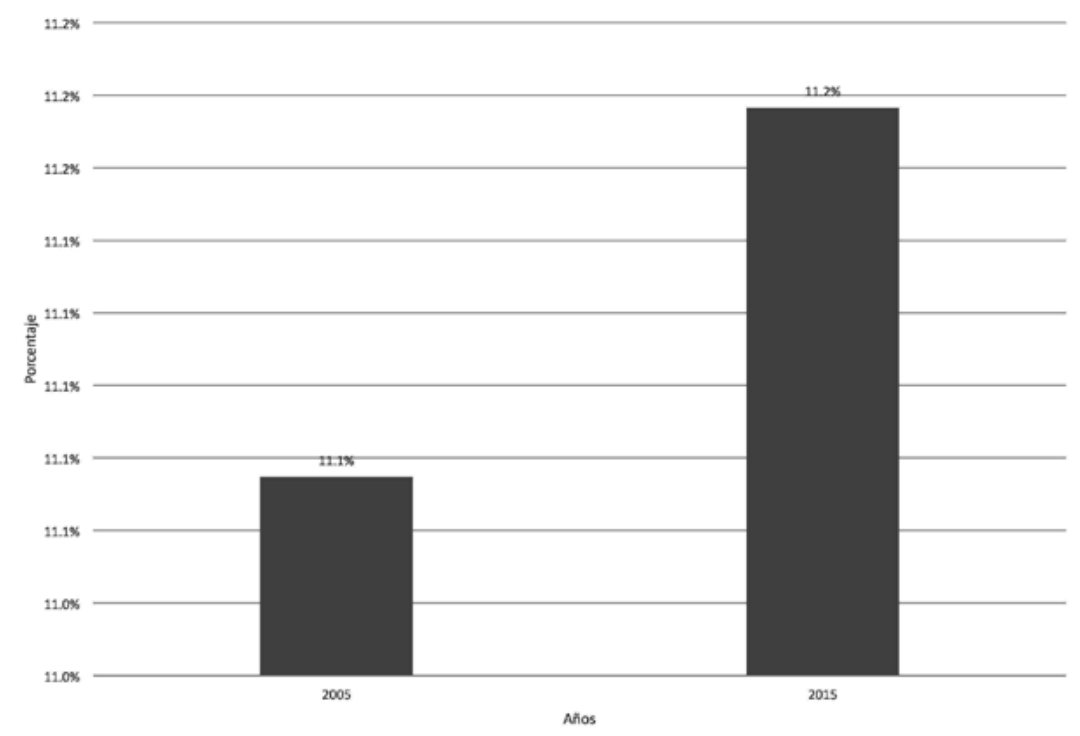

Fuente: elaboración propia con base en datos proporcionados por el DANE.

Para sintetizar los cambios en el mercado laboral en Bogotá, se diseñó la siguiente tabla, la cual relaciona los indicadores obtenidos por la ciudad en 2005 y 2015, junto con sus cambios porcentuales.

Tabla 4. Resumen de los principales indicadores laborales en Bogotá 2005-2015

\begin{tabular}{|l|c|c|c|}
\hline \multicolumn{1}{|c|}{ Concepto } & $\mathbf{2 0 0 5}$ & $\mathbf{2 0 1 5}$ & Diferencia \% \\
\hline \% población en edad de trabajar & $78,5 \%$ & $81,7 \%$ & $3,3 \%$ \\
\hline TGP & $66,4 \%$ & $71,6 \%$ & $5,2 \%$ \\
\hline TO & $57,7 \%$ & $65,4 \%$ & $7,6 \%$ \\
\hline TD & $13,1 \%$ & $8,7 \%$ & $-4,3 \%$ \\
\hline Tasa de subempleo objetivo & $11,1 \%$ & $11,2 \%$ & $0,1 \%$ \\
\hline
\end{tabular}

Fuente: elaboración propia con base en datos proporcionados por el DANE.

\section{Metodología y resultados}

Para determinar los cambios en los factores que componen la estructura del mercado laboral en la ciudad de Bogotá en el período de análisis, se utilizó como referencia el modelo planteado por Tenjo y Ribero (1998), el cual plantea una ecuación de participación 
cuya variable dependiente es una dummy compuesta por " 1 " si el individuo participa en el mercado laboral o "0" si no participa.

Para este estudio se tomó como población objetivo la población en edad de trabajar $(\mathrm{PET})^{5}$. Se construyó una variable dicótoma a la cual se le asignó el valor de "1" cuando el individuo pertenece a la población económicamente activa ${ }^{6}$ (PEA) y " 0 ” cuando pertenece a la población económicamente inactiva ${ }^{7}(\mathrm{PEI})$. Como se sabe, este estudio analiza los determinantes del mercado laboral en Bogotá; para efectos del análisis, se tomó el área metropolitana de Bogotá, la cual está compuesta por los municipios de Bojacá, Cajicá, Chía, Cogua, Cota, El Rosal, Facatativá, Funza, Gachancipá, La Calera, Madrid, Mosquera, Nemocón, Soacha, Sibaté, Sopó, Subachoque, Tabio, Tenjo, Tocancipá y Zipacón.

El modelo que se estimó para este estudio es un modelo de respuesta cualitativa tipo Logit $^{8}$, cuya estructura general es la siguiente:

$$
P_{i}=\frac{1}{1+e^{-}\left(\beta_{0}+\beta_{1} X\right)}(1)
$$

En la fórmula, Pi representa la probabilidad condicional de que ocurra la situación representada por la variable dependiente $\mathrm{Yi}$, lo cual es igual al valor esperado, $\mathrm{E}(\mathrm{Yi}=1 \mid \mathrm{Xi})$. Si se modifica la ecuación (1) y se remplaza la función de regresión por Z, se obtiene la siguiente expresión:

$$
P_{i}=\frac{1}{1+e^{-\mathrm{Zt}}}=\frac{e^{z}}{1+e^{\mathrm{Z}}}
$$

5 La PET está compuesta por los individuos mayores de 12 años que habitan las zonas urbanas y por los menores de 10 años que habitan las zonas rurales.

6 También se le llama fuerza laboral y está conformada por las personas en edad de trabajar que trabajan o están buscando empleo.

7 Comprende todas las personas en edad de trabajar (10 años y más) que en la semana de referencia no participaron en la producción de bienes y servicios porque no necesitaban, no pudieron o no estaban interesadas en tener actividad remunerada. A este grupo pertenecen las personas que son exclusivamente:

- Estudiantes

- Amas de casa

- Pensionados

- Jubilados

- Rentistas

- Incapacitados permanentes para trabajar

- Personas a las que no les llama la atención o creen que no vale la pena trabajar.

- Otros incluidos dentro de la población en edad de trabajar (PET).

8 Es un modelo de respuesta cualitativa, el cual maneja la función logística como base, con el objeto de que las probabilidades calculadas se encuentren entre los valores de " 0 " y " 1 ". 
La ecuación (2) representa lo que se conoce como la función de distribución logística (acumulativa) $^{9}$. Para realizar la estimación de un modelo Logit, se puede realizar una transformación algebraica, la cual se muestra a continuación:

$$
1-P_{i}=\frac{1}{1+e^{\mathrm{z}}}
$$

Por consiguiente, si se opera la expresión anterior, se puede obtener la siguiente expresión:

$$
P_{i}=\frac{1}{1+e-\left(\beta_{0}+\beta_{1} X\right)}
$$

Como se puede observar en la ecuación (5), $\left(P_{1} / 1-P_{\mathrm{t}}\right)$ es la razón de las probabilidades de tener éxito según el evento analizado. Calculando los logaritmos naturales de la expresión anterior, se obtiene:

$$
L_{\mathrm{i}}=\operatorname{In}\left(\frac{P_{1}}{1-P_{\mathrm{t}}}\right)=z=\beta_{0}+\beta_{1} X_{\mathrm{i}}
$$

En la ecuación anterior, L es el logaritmo de la razón de las probabilidades, el cual no es solo lineal en $\mathrm{X}$, sino también - desde el punto de vista de estimación- lineal en los parámetros.

Para aplicar este modelo al estudio, se planteó la siguiente función, la cual tiene por objetivo determinar los cambios en los determinantes de la estructura laboral en la ciudad de Bogotá, comparando los períodos 2005 y 2015:

$$
\begin{aligned}
L_{i}= & P E A=\beta_{0}+\beta_{1} \text { riqueza }+\beta_{2} \text { edad }+\beta_{3} \text { experiencia }+\beta_{4} \text { escolaridad }+\beta_{5} \text { mujer } \\
& +\beta_{6} \text { casado }+\beta_{7} \text { viudo }+\beta_{8} \text { separado }+\beta_{9} \text { soltero }+\beta_{10} \text { estudia }+\mu_{i}(6)
\end{aligned}
$$

En la tabla 5 se describen las variables utilizadas en el modelo.

Tabla 5. Descripción de las variables utilizadas en la estimación de los modelos

\begin{tabular}{|l|c|c|c|}
\hline \multicolumn{1}{|c|}{ Variable } & Tipo & Rol & \multicolumn{1}{c|}{ Descripción } \\
\hline PEA & Dicotómica & Dependiente & $\begin{array}{l}\text { Determina cuáles de los individuos } \\
\text { pertenecientes a la PET forman parte de la PEA } \\
\text { y se les asigna el código de 1, mientras que los } \\
\text { pertenecientes a la PEI se identifican con } 0 .\end{array}$ \\
\hline Riqueza & Cuantitativa & Explicativa & $\begin{array}{l}\text { Es toda la riqueza del individuo tomando todos } \\
\text { los ingresos que genera. }\end{array}$ \\
\hline
\end{tabular}

9 El modelo logístico es muy común en análisis de fenómenos de crecimiento, como población, PNB, oferta monetaria, etc. Para conocer los detalles teóricos y prácticos de los modelos Logit y Probit, véase J. S. Cramer (1991) y G. S. Maddala, op. cit. 


\begin{tabular}{|c|c|c|c|c|c|}
\hline Variable & Tipo & Rol & \multicolumn{3}{|c|}{ Descripción } \\
\hline Edad & Cuantitativa & Explicativa & \multicolumn{3}{|c|}{ Es la edad de años cumplidos del individuo. } \\
\hline Experiencia & Cuantitativa & Explicativa & \multicolumn{3}{|c|}{ Es la edad al cuadrado. } \\
\hline Escolaridad & Cuantitativa & Explicativa & \multicolumn{3}{|c|}{$\begin{array}{l}\text { Son los años que el individuo ha dedicado a } \\
\text { estudiar. }\end{array}$} \\
\hline Mujer & Dicotómica & Explicativa & \multicolumn{3}{|c|}{$\begin{array}{l}\text { Identifica la proporción de la población } \\
\text { compuesta por mujeres con } 1 \text { y se le asigna el } \\
\text { código de } 0 \text { a no mujeres. }\end{array}$} \\
\hline Casado & Dicotómica & Explicativa & \multicolumn{3}{|c|}{$\begin{array}{l}\text { Identifica el estado civil de casado con el código } \\
1 \text { y los demás estados con } 0 .\end{array}$} \\
\hline Viudo & Dicotómica & Explicativa & \multicolumn{3}{|c|}{$\begin{array}{l}\text { Identifica el estado civil de viudo con el código } 1 \\
\text { y los demás estados con } 0 .\end{array}$} \\
\hline Separado & Dicotómica & Explicativa & \multicolumn{3}{|c|}{$\begin{array}{l}\text { Identifica el estado civil de separado con el } \\
\text { código } 1 \text { y los demás estados con } 0 .\end{array}$} \\
\hline Soltero & Dicotómica & Explicativa & \multicolumn{3}{|c|}{$\begin{array}{l}\text { Identifica el estado civil de soltero con el código } \\
1 \text { y los demás estados con } 0 .\end{array}$} \\
\hline Estudia & Dicotómica & Explicativa & \multicolumn{3}{|c|}{$\begin{array}{l}\text { Le asigna el código de } 1 \text { a la persona que está } \\
\text { estudiando en el período de referencia y } 0 \text { a la } \\
\text { que no. }\end{array}$} \\
\hline \multicolumn{6}{|c|}{ Fuente: elaboración propia. } \\
\hline \multirow{2}{*}{\multicolumn{6}{|c|}{$\begin{array}{l}\text { A continuación, se presentan los resultados obtenidos en la estimación de los modelos } \\
\text { para los períodos } 2005 \text { y } 2015 \text {, y se muestran las variables que son y no son significativas } \\
\text { al } 5 \% \text { de significancia. }\end{array}$}} \\
\hline & & & & & \\
\hline Variable & 2005 & 2015 & Variable & 2005 & 2015 \\
\hline Constante & $(-) \mathrm{S}$ & $(-) \mathrm{S}$ & Casado & $(-) \mathrm{S}$ & $(-) \mathrm{NS}$ \\
\hline Riqueza & $(+) \mathrm{S}$ & $(+) S$ & Viudo & $(-) \mathrm{S}$ & $(+) \mathrm{NS}$ \\
\hline Edad & $(+) \mathrm{S}$ & $(+) \mathrm{S}$ & Separado & $(+) \mathrm{NS}$ & $(+) \mathrm{NS}$ \\
\hline Experiencia & $(-) \mathrm{S}$ & $(-) \mathrm{S}$ & Soltero & $(+) \mathrm{NS}$ & $(+) \mathrm{S}$ \\
\hline Escolaridad & $(+) \mathrm{S}$ & $(+) \mathrm{S}$ & Estudia & $(-) \mathrm{S}$ & $(-) \mathrm{S}$ \\
\hline Mujer & $(-) S$ & $(-) \mathrm{S}$ & & & \\
\hline
\end{tabular}

Fuente: cálculos propios.

10 +: Coeficiente positivo, -: Coeficiente negativo, S: Coeficiente estadísticamente significativo al $10 \%$ de significancia, NS: Coeficiente no estadísticamente significativo al $10 \%$ de significancia. 
$\mathrm{Al}$ analizar los determinantes de la estructura del mercado laboral en Bogotá, se puede determinar que los coeficientes en su gran mayoría son significativos, sin embargo, al analizar el estado civil, se puede observar un cambio entre 2005 y 2015, ya que para 2005, los estados civiles separado y soltero no tenían significancia en la probabilidad de pertenecer a la fuerza de trabajo; sin embargo, para 2015 se notó un cambio en la significancia, ya que los estados que perdieron importancia al momento de pertenecer a la fuerza de trabajo fueron casado y viudo. En este contexto, el ser soltero pasa de ser un factor no relevante a uno relevante al momento de pertenecer a la fuerza de trabajo en la actualidad.

Tabla 7. Análisis comparativo de la participación laboral para 2005 y 2015. (Ver anexo 2)

\begin{tabular}{|l|c|c|}
\hline & \multicolumn{2}{|c|}{ Efecto marginal } \\
\hline \multicolumn{1}{|c|}{ Variable } & $\mathbf{2 0 0 5}$ & $\mathbf{2 0 1 5}$ \\
\hline Riqueza & $1,30 \mathrm{e}-07$ & 0,0172 \\
\hline Edad & 0,0686 & 0,0081 \\
\hline Experiencia & $-0,0008$ & $-0,00009$ \\
\hline Escolaridad & 0,0061 & 0,0037 \\
\hline Mujer & $-0,1983$ & $-0,0239$ \\
\hline Casado & $-0,1316$ & $-0,0129$ \\
\hline Viudo & $-0,1769$ & 0,0114 \\
\hline Separado & 0,0685 & 0,0124 \\
\hline Soltero & 0,0432 & 0,0191 \\
\hline Estudia & $-0,4211$ & $-0,0764$ \\
\hline
\end{tabular}

Fuente: cálculos propios.

La tabla 7 evidencia que, en 2005, el determinante que más incidió en reducir la probabilidad de participar en el mercado laboral fue que la persona estudiara $(42,11 \%)$; sin embargo, esta probabilidad se redujo al 7,64 \% en 2015, lo cual puede ser explicado porque la población estudiantil ha empezado a ejercer también la actividad laboral. En segundo lugar, la población femenina ceteris paribus fue otro factor que redujo la probabilidad de participar en la fuerza laboral, con un 19,83 \% en 2005, mientras que para 2015 se observó que esta probabilidad disminuyó significativamente, llegando a ser solo del 2,3\%. Por su lado, la edad aumentó en mayor medida la probabilidad de formar parte de la fuerza laboral en 2005, en un 6,86 \%; sin embargo, para 2015 esta probabilidad se redujo a solo $0,81 \%$. También se puede inferir que las probabilidades de todas las variables se redujeron de 2005 a 2015, lo que se traduce en que los determinantes 
positivos de la participación laboral disminuyeron la probabilidad de participar en la fuerza de trabajo para el año 2015.

\section{A manera de discusión}

De acuerdo con los resultados de las estimaciones econométricas, se puede argüir que las variables riqueza, edad, experiencia, escolaridad, mujer y estudia explican estadísticamente la fuerza laboral en Bogotá en los dos años de estudio, mientras que las personas separadas no resultaron determinantes al momento de participar en el mercado de trabajo, tanto para el año 2005 como para el 2015.

También se pueden evidenciar los cambios presentes en los determinantes de la participación laboral; de esta manera, las personas casadas y las viudas fueron factores determinantes en la fuerza de trabajo en 2005, pero en 2015 dejaron de serlo. Por su parte, el estado civil soltero no resultó determinante en 2005, pero sí lo fue en el 2015.

Finalmente, se resalta la reducción significativa de la probabilidad de que los estudiantes y las mujeres no hayan participado en el mercado laboral del año 2005 al 2015, lo cual evidencia que esta población ha decidido emplearse o buscar empleo. En este sentido, las estrategias de política de empleo deben concentrar esfuerzos para mejorar las condiciones de trabajo de este tipo de población, incentivando a las empresas a contratar a la población estudiantil y femenina.

\section{Referencias}

Alarco, G., Lora, E. y Orellana, S. (1990). Técnicas de medición económica. Lima: Centro de Economía Aplicada, Consejo Nacional de Ciencia y Tecnología.

Arango, L. E. y Posada, C. E. (2001). El desempleo en Colombia (Borradores de Economía n.o 176). Bogotá: Banco de la República. Recuperado de goo.gl/nxF22Z . (2003). La participación laboral en Colombia. Coyuntura Social, 28, 63-83. Recuperado de goo.gl/L4AaRw

Arango, L. E., Posada, C. E. y Charry, A. (2003). La participación laboral en Colombia según la nueva encuesta: ¿cambian sus determinantes? (Borradores de Economía n.o 250). Bogotá: Banco de La República. Recuperado de goo.gl/um5c9k

Borjas, G. (2000). Labor Economics (2.a ed.). Boston y Londres: Irwin-McGraw-Hill. 
Blundell, R. y Thomas, M. (1999). Labor supply: A review of alternative approaches. En O. Ashenfelter y D. Card (Eds.), Handbook of Labor Economics (volumen 3, parte A) (pp. 1559-1695). North Holland: Elsevier Science. Recuperado de goo.gl/ogZlwL

Cramer, J. S. (1991). The Logit model for economists. Londres: Edward Arnold.

Chávez Muñoz, N. M., Velásquez Mantilla, O. D. y Mateus Tovar, M. A. (2011). Cambios estructurales en la participación laboral en Colombia desde 1984-2008: un análisis econométrico del mercado laboral urbano para la generación de políticas de empleo. Revista CIFE, 13(18), 79-100. DOI: 10.15332/s2248-4914.2011.0018.02

Departamento Administrativo Nacional de Estadística - DANE. (2005). Encuesta Continua de Hogares. Bogotá: DANE. . (2015). Gran Encuesta Integrada de Hogares. Bogotá: DANE.

Deaton, A. y Muellbauer,J. (1980). Economics and consumer behavior. Cambridge: Cambridge University Press.

Farné, S. (1994). La oferta de trabajo urbano en Colombia, 1976-1992. Desarrollo y Sociedad, 34, 149-77.

Gilbert, A. (1997). Employment and poverty during economic restructuring: The case of Bogotá, Colombia. Urban Studies, 34(7), 1047-1070.

Heckman, J. (1993). What has been learned about labor supply in the past twenty years? American Economic Review, 83(2), 116-121. Recuperado de goo.gl/3jPS5H

Isaza, J. (2002). Women workers in Bogota's informal sector: Gendered impact of structural adjustment policies -SAP-in the 1990s. Planeación y Desarrollo, 33(3-4).

Kaufman, B. y Hotchkiss, J. (2000). The economics of labor markets (5.a ed.). San Diego, CA: Hartcourt College Publishers.

López, H. (2001). Características y determinantes de la oferta laboral colombiana y su relación con la dinámica del desempleo. En M. Urrutia (Ed.), Empleo y economía. Bogotá: Banco de la República.

McConnell, G. y Brue, S. (2003). Economía laboral contemporánea (Contemporary Labor Economics) (6.a ed.) (Trad. E. Rabasco y L. Toharia). Madrid: McGraw-Hill.

Pecable, J. (1986). Labor supply of men: A survey. En O. Ashenfelter y R. Layard (Eds.), Handbook of labor economics (Vol. 1) (Cap. 1). North Holland: Elsevier Science. Recuperado de goo.gl/TKi6Lk 
Romero, D. (2006). Determinantes de la participación laboral en Bogotá 2001-2004. Recuperado de goo.gl/d2Q5gU

Santamaría, M. y Rojas, N. (2001). La participación laboral: ¿qué ha pasado y qué podemos esperar? (Serie Archivos de Economía n.o 146). Bogotá: Departamento Nacional de Planeación.

Suárez, Á. y Buriticá, A. (2002). Empalme de las series de tasa de desempleo, ocupación y participación entre la encuesta transversal y la encuesta continua. Bogotá: DANE.

Tenjo, J. y Ribero, R. (1998). Participación, desempleo y mercados laborales en Colombia (Serie Archivos de Economía n.o 81). Bogotá: Departamento Nacional de Planeación. 


\section{Anexos}

\subsection{Anexo 1}

Resultados del modelo Logit en Bogotá para el año 2005

\begin{tabular}{|c|c|c|c|c|}
\hline \multicolumn{5}{|l|}{ Logit 2005} \\
\hline \multirow[t]{3}{*}{ Logistic regression } & & Number of obs & $=$ & 2464 \\
\hline & & $\mathrm{LR}$ chi2 $(10)$ & $=$ & 1249.71 \\
\hline & & Prob $>$ chi2 & $=$ & 0.0000 \\
\hline Log likelihood & -938.38393 & Pseudo R2 & $=$ & 0.3997 \\
\hline
\end{tabular}

\begin{tabular}{|l|c|c|c|c|c|c|}
\hline \multicolumn{1}{|c|}{ pea } & Coef. & Std. Err. & $\mathbf{z}$ & \multicolumn{2}{c|}{$\mathbf{P}>\mathbf{z} \mid$} & \multicolumn{2}{|c|}{ [95\% Conf. Interval] } \\
\hline riqueza & $6.54 \mathrm{e}-07$ & $1.31 \mathrm{e}-07$ & 4.99 & 0.000 & $3.97 \mathrm{e}-07$ & $9.11 \mathrm{e}-07$ \\
\hline edad & .3462226 & .0234372 & 14.77 & 0.000 & .3002866 & .3921587 \\
\hline experiencia & -.0044274 & .0002739 & -16.16 & 0.000 & -.0049643 & -.0038906 \\
\hline escolaridad & .0310281 & .0174737 & 1.78 & 0.076 & -.0032197 & .0652758 \\
\hline mujer & -1019661 & .1259255 & -8.10 & 0.000 & -1266471 & -.7728518 \\
\hline casado & -.6225583 & .184155 & -3.38 & 0.001 & -.9834954 & -.2616212 \\
\hline viudo & -.787249 & .3380417 & -2.33 & 0.020 & -1449799 & -.1246994 \\
\hline separado & .3719484 & .2582587 & 1.44 & 0.150 & -.1342294 & .8781262 \\
\hline soltero & .2209673 & .1917435 & 1.15 & 0.249 & -.154843 & .5967776 \\
\hline estudia & -1.872499 & .1757306 & -10.66 & 0.000 & -2216925 & -1528074 \\
\hline cons & -4.050935 & .4742027 & -8.54 & 0.000 & -4980355 & -3121515 \\
\hline
\end{tabular}

Fuente: cálculos propios. 
Resultados del modelo Logit en Bogotá para el año 2015

\begin{tabular}{|c|c|c|c|c|c|c|}
\hline \multicolumn{7}{|l|}{ Logit 2015} \\
\hline \multicolumn{3}{|c|}{ Logistic regression } & \multicolumn{2}{|r|}{ Number of obs } & $=$ & 2463 \\
\hline & & & \multicolumn{2}{|r|}{ LR chi2(10) } & $=$ & 2051.39 \\
\hline & & & \multicolumn{2}{|r|}{ Prob > chi2 } & $=$ & 0.0000 \\
\hline Log likelihood & $=$ & -42361748 & \multicolumn{2}{|r|}{ Pseudo R2 } & $=$ & 0.7077 \\
\hline pea & Coef. & Std. Err. & $\mathbf{z}$ & $P>|z|$ & \multicolumn{2}{|c|}{ [95\% Conf. Interval] } \\
\hline riqueza & .4253369 & .0247442 & 17.19 & 0.000 & .3768391 & .4738348 \\
\hline edad & .2004714 & .0311997 & 6.43 & 0.000 & .1393211 & .2616218 \\
\hline experiencia & -.0023896 & .0003329 & -7.18 & 0.000 & -.0030422 & -.0017371 \\
\hline escolaridad & .0931671 & .0212468 & 4.38 & 0.000 & .0515241 & .1348102 \\
\hline casado & -.2942803 & .2991372 & -0.98 & 0.325 & -.8805784 & .2920179 \\
\hline separado & .3404824 & .3346567 & 1.02 & 0.309 & -.3154326 & .9963974 \\
\hline viudo & .3210414 & .4654668 & 0.69 & 0.490 & -.5912569 & 1.23334 \\
\hline soltero & .4949769 & .3030605 & 1.63 & 0.102 & -.0990107 & 1.088965 \\
\hline mujer & -.5906482 & .189313 & -3.12 & 0.002 & -.961695 & -.2196015 \\
\hline estudia & -1.283574 & .2836237 & -4.53 & 0.000 & -1.839466 & -.7276815 \\
\hline _cons & -4663057 & .7269332 & -6.41 & 0.000 & -608782 & -3.238294 \\
\hline
\end{tabular}

Fuente: cálculos propios. 


\subsection{Anexo 2}

Efectos marginales del modelo Logit en Bogotá para el año 2005

\begin{tabular}{|c|c|c|c|c|c|c|c|}
\hline \multicolumn{8}{|c|}{ Marginal effects after logit } \\
\hline \multirow[t]{2}{*}{$\mathrm{y}$} & $=$ & \multicolumn{6}{|c|}{$\operatorname{Pr}($ pea) (predict) } \\
\hline & $=$ & \multicolumn{6}{|l|}{.72722363} \\
\hline variable & $d y / d x$ & Std. Err. & $\mathbf{z}$ & $\mathbf{P}>|\mathbf{z}|$ & \multicolumn{2}{|c|}{ [95\% C.I.] } & $\mathbf{x}$ \\
\hline riqueza & .0172733 & .00000 & 5.15 & 0.000 & $8.0 \mathrm{e}-08$ & $1.8 \mathrm{e}-07$ & 450416 \\
\hline edad & .06868 & .00499 & 13.75 & 0.000 & .058893 & .078467 & 37.2731 \\
\hline expericia & -.0008783 & .00006 & -14.89 & 0.000 & -.000994 & -.000763 & 1698.9 \\
\hline escolaridad & .006155 & .00347 & 1.77 & 0.076 & -.000653 & .012963 & 10.9168 \\
\hline mujer* & -.1983078 & .02369 & -8.37 & 0.000 & -.24474 & -.151876 & .525568 \\
\hline casado* & -.1316371 & .04083 & -3.22 & 0.001 & -.211659 & -.051616 & .239854 \\
\hline viudo* & -.1769694 & .08256 & -2.14 & 0.032 & -.338775 & -.015164 & .051948 \\
\hline separado* & .0685823 & .04393 & 1.56 & 0.119 & -.017528 & .154692 & .096997 \\
\hline soltero* & .0432968 & .03711 & 1.17 & 0.243 & -.029446 & .116039 & .382305 \\
\hline estudia* & -.4211667 & .03836 & -10.98 & 0.000 & -.496346 & -.345987 & .202516 \\
\hline
\end{tabular}

$\left.{ }^{*}\right) \mathrm{dy} / \mathrm{dx}$ is for discrete change of dummy variable from 0 to 1

Fuente: cálculos propios.

Efectos marginales del modelo Logit en Bogotá para el año 2015

\begin{tabular}{|c|c|c|c|c|c|c|c|}
\hline \multicolumn{8}{|c|}{ Marginal effects after logit } \\
\hline \multirow[t]{2}{*}{ y } & $=$ & \multicolumn{6}{|c|}{$\operatorname{Pr}($ pea $)$ (predict) } \\
\hline & $=$ & \multicolumn{6}{|l|}{.95759048} \\
\hline variable & $d y / d x$ & Std. Err. & $\mathbf{z}$ & $P>|z|$ & \multicolumn{2}{|c|}{ [95\% C.I. $]$} & $\mathbf{X}$ \\
\hline riqueza & .0172733 & .00256 & 6.74 & 0.000 & .01225 & .022297 & 90,157 \\
\hline edad & .0081413 & .00199 & 4.10 & 0.000 & .004245 & .012038 & 38.9342 \\
\hline expericiaa & -.000097 & .00002 & -4.30 & 0.000 & -.000141 & -.000053 & 1847.31 \\
\hline escolaridad & .0037836 & .00109 & 3.49 & 0.000 & .001657 & .00591 & 10.0459 \\
\hline casado* & -.0129821 & .01453 & -0.89 & 0.372 & -.041466 & .015502 & .20138 \\
\hline separado*| & .0124505 & .01125 & 1.11 & 0.268 & -.009598 & .034499 & .153065 \\
\hline viudo*| & .0114384 & .01453 & 0.79 & 0.431 & -.017031 & .039908 & .045879 \\
\hline soltero*| & .0191673 & .01181 & 1.62 & 0.105 & -.00398 & .042314 & .377994 \\
\hline mujer*| & -.0239001 & .00861 & -2.78 & 0.006 & -.040779 & -.007022 & .527 \\
\hline estudia*| & -.076401 & .02633 & -2.90 & 0.004 & -.128005 & -.024797 & .206253 \\
\hline
\end{tabular}

$(*) \mathrm{dy} / \mathrm{dx}$ is for discrete change of dummy variable from 0 to 1 